\title{
ON THE ABSOLUTE CESARO SUMMABILITY OF FOURIER SERIES
}

\author{
HIROSHI HIROKAWA
}

1. Let $f(t)$ be an even integrable function with period $2 \pi$ and let

$$
\begin{array}{rlrl}
f(t) & \sim \sum_{n=1}^{\infty} a_{n} \cos n t, \\
\Phi_{\alpha}(t) & =\int_{0}^{t}(t-u)^{\alpha-1} f(u) d u, & \alpha>0 .
\end{array}
$$

L. S. Bosanquet [2] proved the following:

ThEOREM A. If $\Phi_{\alpha}(t) / t^{\alpha}$ is of bounded variation in $(0, \pi)$, then, for $\gamma>\alpha$, the Fourier series of $f(t)$ at $t=0$ is evaluable $|C, \gamma|$, and conversely, if the Fourier series of $f(t)$ at $t=0$ is evaluable $|C, \gamma|$, then, for $\alpha>\gamma+1, \Phi_{\alpha}(t) / t^{\alpha}$ is of bounded variation in $(0, \pi)$.

Earlier, L. S. Bosanquet [1] proved the following:

THEOREM B. If $\Phi_{\alpha}(t) / t^{\alpha}$ is of bounded variation in an interval to the right of $t=0$, then the Fourier series of $f(t)$ at $t=0$ is evaluable $(C, \alpha-1)$ when $\alpha \geqq 1$.

The purpose of this paper is to establish the following:

TheOREM. If the Fourier series of $f(t)$ at $t=0$ is evaluable $|C, \alpha|$ to zero, then $\Phi_{\alpha}(t)=o\left(t^{\alpha}\right)$ when $\alpha \geqq 1$.

This theorem was implicitly proved by N. Obreschkoff [4, Satz 2] when $\alpha=1$.

2. Preliminary lemmas.

LEMMA 1. Let a series $\sum_{n=1}^{\infty} b_{n}$ be convergent and let

Then

$$
c_{n}=\sum_{v=n}^{\infty} b_{v}
$$

$$
\sum_{v=n}^{m} a_{\nu} b_{\nu}=a_{n} c_{n}-a_{m} c_{m+1}-\sum_{v=n}^{m-1} c_{\nu+1} \Delta a_{\nu}
$$

Received by the editors November 16, 1959 and, in revised form, July 9, 1960 and January 30, 1961. 
where $\Delta a_{v}=a_{v}-a_{v+1}$. In particular, if $a_{m} c_{m+1} \rightarrow 0$ as $m \rightarrow \infty$,

$$
\sum_{v=n}^{\infty} a_{\nu} b_{\nu}=a_{n} c_{n}-\sum_{n=n}^{\infty} c_{n+1} \Delta a_{\nu}
$$

Proof is obvious.

Throughout this paper, let

$$
\begin{aligned}
{[x] } & \text { be the greatest integer less than } x, \\
\beta & =[\alpha], \\
A_{n}^{\gamma} & =\left(\begin{array}{c}
\gamma+n \\
n
\end{array}\right)=\frac{\Gamma(n+\gamma+1)}{\Gamma(n+1) \Gamma(\gamma+1)}, \\
s_{n}^{\gamma} & =\sum_{v=0}^{n} A_{n-r}^{\gamma} a_{v}, \\
\phi(n, t) & =\int_{0}^{t}\left(t^{2}-u^{2}\right)^{\alpha-1} \cos n u d u, \\
\Delta^{k} \phi(n, t) & =\Delta^{k-1} \phi(n, t)-\Delta^{k-1} \phi(n+1, t), \\
\psi(\mu, t) & =2^{\mu} \Gamma(\mu+1) t^{-\mu} J_{\mu}(t),
\end{aligned}
$$

where $J_{\mu}(t)$ is the Bessel function of order $\mu, \mu>-1 / 2$.

Lemma 2. For $\alpha>0$ and $k=0,1,2, \cdots$,

$$
\Delta^{k} \phi(n, t)=O\left(n^{-\alpha} t^{\alpha+k-1}\right) .
$$

Proof. Since

$$
\psi\left(\alpha-\frac{1}{2}, n t\right)=\frac{2 \Gamma(\alpha+1 / 2)}{\Gamma(1 / 2) \Gamma(\alpha)} t^{1-2 \alpha} \phi(n, t),
$$

we have the lemma by a known result. (See K. Chandrasekharan and O. Szász [3, pp. 716 and 728].)

Lemma 3. For $\alpha>0$ and $j=0,1,2, \cdots$, such that $\alpha+j \geqq 1$,

$$
\sum_{n=\nu}^{\infty} A_{n-\nu}^{\beta-\alpha} \Delta^{\beta+j} \phi(n, t)=O\left(\nu^{-\alpha} t^{2 \alpha-2+j}\right) .
$$

Proof. The lemma in which $\alpha$ is an integer is obvious by (2.3), so that we shall prove the lemma in which $\alpha$ is not an integer. Putting $\rho=\left[t^{-1}\right]$ and using (2.2) and (2.3), 


$$
\begin{aligned}
\sum_{n=\nu}^{\infty} A_{n-\nu}^{\beta-\alpha} \Delta^{\beta+j} \phi(n, t)= & \left(\sum_{n=\nu}^{++\rho-1}+\sum_{n=\nu+\rho}^{\infty}\right) \\
= & O\left(t^{\alpha+\beta+j-1} \sum_{n=\nu}^{\nu+\rho-1} A_{n-\nu}^{\beta-\alpha} n^{-\alpha}\right)+A_{\rho}^{\beta-\alpha} \Delta^{\beta+j-1} \phi(\nu+\rho, t) \\
& +\sum_{n=\nu+\rho}^{\infty} A_{n-\nu+1}^{\beta-\alpha-1} \Delta^{\beta+j-1} \phi(n+1, t) \\
= & O\left(\nu^{-\alpha} t^{\alpha+\beta+j-1} A_{\rho-1}^{\beta-\alpha+1}\right)+O\left(\nu^{-\alpha} t^{\alpha+\beta+j-2} \cdot \rho^{\beta-\alpha}\right) \\
& +O\left(t^{\alpha+\beta+j-2} \sum_{n-\nu+\rho}^{\infty}(n-\nu+1)^{\beta-\alpha-1} n^{-\alpha}\right) \\
= & O\left(\nu^{-\alpha} t^{2 \alpha+j-2}\right)+O\left(\nu^{-\alpha \alpha+\beta+j-2} \cdot \rho^{\beta-\alpha}\right)=O\left(v^{-\alpha} t^{2 \alpha-2+j}\right)
\end{aligned}
$$

which is the required result.

LEMMA 4. Let $\alpha \geqq 1$. Then, for any $m$ and $t>0$,

$$
U_{m}(t) \equiv \sum_{\nu=1}^{m} A_{\nu}^{\alpha} \sum_{n=\nu}^{\infty} A_{n-\nu}^{\beta-\alpha} \Delta^{\beta+2} \phi(n, t)=O\left(t^{2 \alpha-1}\right) .
$$

Proof. If $m t \leqq 1$, then, using (2.5) for $j=2$,

$$
U_{m}(t)=O\left(\sum_{\nu=1}^{m} A_{\nu}^{\alpha} \cdot \nu^{-\alpha} \cdot t^{2 \alpha}\right)=O\left(m t^{2 \alpha}\right)=O\left((m t) \cdot t^{2 \alpha-1}\right)=O\left(t^{2 \alpha-1}\right)
$$

Therefore we shall prove (2.6) when $m t>1$. Putting $\rho=\left[t^{-1}\right]$, we write

$$
U_{m}(t)=\left(\sum_{r=1}^{\rho-1}+\sum_{v=\rho}^{m}\right)=U_{m 1}(t)+U_{m 2}(t)
$$

where $U_{m 1}(t)=O\left(t^{2 \alpha-1}\right)$ by (2.7). Since, for $j=0,-1$, using (2.5),

$$
\begin{aligned}
& \sum_{\nu=k}^{\infty} \sum_{n=\nu}^{\infty} A_{n-\nu}^{\beta-\alpha} \Delta^{\beta+2+j} \phi(n, t) \\
& =\lim _{p \rightarrow \infty} \sum_{\nu=k}^{p}\left(\sum_{n=0}^{\infty} A_{n}^{\beta-\alpha} \Delta^{\beta+1+j} \phi(n+\nu, t)-\sum_{n=0}^{\infty} A_{n}^{\beta-\alpha} \Delta^{\beta+1+j} \phi(n+\nu+1, t)\right) \\
& =\lim _{p \rightarrow \infty}\left(\sum_{n=0}^{\infty} A_{n}^{\beta-\alpha} \Delta^{\beta+1+j} \phi(n+k, t)-\sum_{n=0}^{\infty} A_{n}^{\beta-\alpha} \Delta^{\beta+1+j} \phi(n+p+1, t)\right) \\
& =\sum_{n=k}^{\infty} A_{n-k}^{\beta-\alpha} \Delta^{\beta+1+j} \phi(n, t),
\end{aligned}
$$


we have, by (2.1) and (2.5),

$$
\begin{aligned}
& U_{m 2}(t)=A_{\rho}^{\alpha} \sum_{n=\rho}^{\infty} A_{n-\rho}^{\beta-\alpha} \Delta^{\beta+1} \phi(n, t)-A_{m}^{\alpha} \sum_{n=m+1}^{\infty} A_{n-m-1}^{\beta-\alpha} \Delta^{\beta+1} \phi(n, t) \\
& +\sum_{\nu=\rho}^{m-1} A_{\nu+1}^{\alpha-1} \sum_{n=\nu+1}^{\infty} A_{n-\nu-1}^{\beta-\alpha} \Delta^{\beta+1} \phi(n, t) \\
& =A_{\rho}^{\alpha} \sum_{n=\rho}^{\infty} A_{n-\rho}^{\beta-\alpha} \Delta^{\beta+1} \phi(n, t)-A_{m}^{\alpha} \sum_{n=m+1}^{\infty} A_{n-m-1}^{\beta-\alpha} \Delta^{\beta+1} \phi(n, t) \\
& +A_{\rho+1}^{\alpha-1} \sum_{n=\rho+1}^{\infty} A_{n-\rho-1}^{\beta-\alpha} \Delta^{\beta} \phi(n, t)-A_{m}^{\alpha-1} \sum_{n=m+1}^{\infty} A_{n-m-1}^{\beta-\alpha} \Delta^{\beta} \phi(n, t) \\
& +\sum_{\nu=\rho}^{m-2} A_{\nu+2}^{\alpha-2} \sum_{n=\nu+2}^{\infty} A_{n-\nu-2}^{\beta-\alpha} \Delta^{\beta} \phi(n, t) \\
& =O\left(\rho^{\alpha} \cdot \rho^{-\alpha} \cdot l^{2 \alpha-1}\right)+O\left(m^{\alpha} \cdot m^{-\alpha} \cdot t^{2 \alpha-1}\right)+O\left(\rho^{\alpha-1} \cdot \rho^{-\alpha} \cdot t^{2 \alpha-2}\right) \\
& +O\left(m^{\alpha-1} \cdot m^{-\alpha} t^{2 \alpha-2}\right)+O\left(t^{2 \alpha-2} \sum_{\nu=\rho}^{\infty} \nu^{\alpha-2} \cdot \nu^{-\alpha}\right) \\
& =O\left(t^{2 \alpha-1}\right)+O\left(m^{-1} \cdot t^{2 \alpha-2}\right)+O\left(\rho^{-1} \cdot t^{2 \alpha-2}\right) \\
& =O\left(t^{2 \alpha-1}\right) \text {, }
\end{aligned}
$$

which is the required result.

LeмmA 5. If the series $\sum_{n=0}^{\infty} a_{n}$ with $a_{0}=0$ is evaluable $|C, \alpha|, \alpha>0$, to zero, then the series $\sum_{n=1}^{\infty} a_{n} \phi(n, t)$ is convergent for any $t>0$ and

$$
\sum_{n=1}^{\infty} a_{n} \phi(n, t)=\sum_{\nu=1}^{\infty} s_{\nu}^{\alpha-1} \sum_{n=\nu}^{\infty} A_{n-\nu}^{\beta-\alpha} \Delta^{\beta+1} \phi(n, t) .
$$

REMARK. It is remarkable that in this lemma the series $\sum_{n=1}^{\infty} a_{n} \cos n t$ need not be a Fourier series.

Proof. The summability $|C, \alpha|$ of the series $\sum_{n=0}^{\infty} a_{n}$ implies that the series $\sum_{n=1}^{\infty} n^{-\alpha}\left|a_{n}\right|$ is convergent, by the well-known theorem. Hence, by (2.3), it follows that the series $\sum_{n=1}^{\infty} a_{n} \phi(n, t)$ is convergent for any $t>0$. By the repeated use of Abel's transformation,

$$
\sum_{n=1}^{m} a_{n} \phi(n, t)=\sum_{n=1}^{m-\beta-1} s_{n}^{\beta} \Delta^{\beta+1} \phi(n, t)+\sum_{j=0}^{\beta} s_{m-j}^{j} \Delta^{j} \phi(m-j, t),
$$

where, by $s_{n}^{\prime}=o\left(n^{\alpha}\right)$ when $0 \leqq j \leqq \alpha$ and (2.3),

$$
\sum_{j=0}^{\beta} s_{m-j}^{j} \Delta^{j} \phi(m-j, t)=o(1) \quad \text { as } m \rightarrow \infty .
$$


Thus

$$
\sum_{n=1}^{\infty} a_{n} \phi(n, t)=\sum_{n=1}^{\infty} s_{n}^{\beta} \Delta^{\beta+1} \phi(n, t)
$$

By the well-known formula

$$
s_{n}^{\beta}=\sum_{\nu=0}^{n} A_{n-\nu}^{\beta-\alpha \alpha-1}=\sum_{\nu=1}^{n} A_{n \rightarrow \nu}^{\beta-\alpha \alpha-1},
$$

we have formally

$$
\begin{aligned}
\sum_{n=1}^{\infty} s_{n}^{\beta} \Delta^{\beta+1} \phi(n, t) & =\sum_{n=1}^{\infty} \Delta^{\beta+1} \phi(n, t) \sum_{\nu=1}^{n} A_{n-\nu}^{\beta-\alpha} s_{\nu}^{\alpha-1} \\
& =\sum_{\nu=1}^{\infty} s_{\nu}^{\alpha-1} \sum_{n=\nu}^{\infty} A_{n \rightarrow \nu}^{\beta-\alpha} \Delta^{\beta+1} \phi(n, t) .
\end{aligned}
$$

Thus, if we may prove that this interchange is legitimate, then the proof is complete. For this purpose, it is sufficient to prove that, for a fixed $t>0$,

$$
\lim _{N \rightarrow \infty} \sum_{\nu=1}^{N} s_{\nu}^{\alpha-1} \sum_{n=N+1}^{\infty} A_{n-\nu}^{\beta-\alpha} \Delta^{\beta+1} \phi(n, t)=0 .
$$

Since, by (2.3),

$$
\sum_{\nu=n}^{\infty} \Delta^{\beta+1} \phi(\nu, t)=\Delta^{\beta} \phi(n, t)
$$

we have (2.8) for an integer $\alpha$, using $s_{n}^{\alpha}=o\left(n^{\alpha}\right)$ and (2.3). Hence we shall prove (2.8) in which $\alpha$ is not an integer. By (2.2), (2.3) and (2.9), for $\nu, 1 \leqq \nu \leqq N$,

$$
\begin{aligned}
\sum_{n=N+1}^{\infty} A_{n-\nu}^{\beta-\alpha} \Delta^{\beta+1} \phi(n, t) & \\
= & A_{N-\nu+1}^{\beta-\alpha} \Delta^{\beta} \phi(N+1, t)+\sum_{n=N+1}^{\infty} A_{n-\nu+1}^{\beta-\alpha-1} \Delta^{\beta} \phi(n+1, t) \\
= & O\left((N-\nu+1)^{\beta-\alpha} N^{-\alpha}\right) .
\end{aligned}
$$

Now the summability $|C, \alpha|$ of the series $\sum_{n=0}^{\infty} a_{n}$ implies

$$
\sum_{\nu=1}^{n}\left|s_{\nu}^{\alpha-1}\right|=O\left(n^{\alpha}\right)
$$

by the series analogue of Rajagopal's Lemma [5, Lemma 10]. Then, let us write, putting $\eta=[N / 2]$, 


$$
\sum_{\nu=1}^{N} s_{\nu}^{\alpha-1} \sum_{n=N+1}^{\infty} A_{n-\nu}^{\beta-\alpha} \Delta^{\beta+1} \phi(n, t)=\left(\sum_{\nu=1}^{n-1}+\sum_{\nu=\eta}^{N}\right)=I_{1}+I_{2},
$$

where, by (2.10) and (2.11),

$$
\begin{aligned}
I_{1} & =O\left(\sum_{\nu=1}^{\eta-1}\left|s_{\nu}^{\alpha-1}\right| \cdot(N-\nu+1)^{\beta-\alpha} \cdot N^{-\alpha}\right) \\
& =O\left(N^{\beta-2 \alpha} \sum_{\nu=1}^{\eta-1}\left|s_{\nu}^{\alpha-1}\right|\right)=O\left(N^{\beta-\alpha}\right)=o(1) .
\end{aligned}
$$

For $I_{2}$, we take an arbitrarily fixed number $L$, but large enough. Then, using (2.10), (2.11) and $s_{n}^{\alpha-1}=o\left(n^{\alpha}\right)$,

$$
\begin{aligned}
I_{2}= & O\left(N^{-\alpha} \sum_{\nu=\eta}^{N}\left|s_{\nu}^{\alpha-1}\right| \cdot(N-\nu+1)^{\beta-\alpha}\right) \\
= & O\left(N^{-\alpha} \sum_{\nu=1}^{N-\eta+1} \mid \begin{array}{c}
\alpha-1 \\
s_{N-\nu+1} \mid \cdot \nu^{\beta-\alpha}
\end{array}\right) \\
= & O\left(N^{-\alpha} \sum_{\nu=1}^{L-1}\left|s_{N-\nu+1}^{\alpha-1}\right| \cdot \nu^{\beta-\alpha}\right)+O\left(N^{-\alpha} \sum_{\nu=L}^{N-\eta+1}\left|s_{N-\nu+1}^{\alpha-1}\right| \cdot \nu^{\beta-\alpha}\right) \\
= & o\left(\sum_{\nu=1}^{L} \nu^{\beta-\alpha}\right)+O\left(N^{-\alpha} \sum_{\nu=1}^{L}\left|s_{N-\nu+1}^{\alpha-1}\right| \cdot L^{\beta-\alpha}\right) \\
& +O\left(N^{-\alpha} \sum_{\nu=1}^{N-\eta+1}\left|s_{N-\nu+1}^{\alpha-1}\right| \cdot(N-\eta+1)^{\beta-\alpha}\right) \\
& +O\left(N^{-\alpha} \sum_{\nu=L}^{N-\eta}\left(\sum_{n=1}^{\nu}\left|s_{N-n+1}^{\alpha-1}\right|\right) \cdot \nu \nu^{\beta-\alpha-1}\right) \\
= & o(1)+O\left(L^{\beta-\alpha}\right)+O\left(N^{\beta-\alpha}\right)+O\left(L^{\beta-\alpha}\right) \\
= & o(1)+O\left(L^{\beta-\alpha}\right) .
\end{aligned}
$$

Since $L$ is arbitrary, we have $I_{2}=o(1)$ by $\beta-\alpha<0$. Thus $(2.8)$ in which $\alpha$ is not an integer is valid.

3. Proof of theorem. The notations in the former paragraphs are used without further explanations. We shall now prove the theorem in which $\alpha$ is not an integer, the remaining part ${ }^{1}$ being obtained by the analogous method. In this case, by the Chandrasekharan and Szász Theorem [3, Theorem 5] for the proof, it is sufficient to prove that

${ }^{1}$ This part is obtained as a corollary of Obreschkoff's Theorem [4, Satz 2] using the following Chandrasekharan and Szász Theorem. This remark is due to Professor G. Sunouchi. 


$$
\Phi_{\alpha}^{*}(t) \equiv \int_{0}^{t}\left(t^{2}-u^{2}\right)^{\alpha-1} f(u) d u=o\left(t^{2 \alpha-1}\right)
$$

Since $\alpha \geqq 1$, we have

$$
\Phi_{\alpha}^{*}(t)=\sum_{n=1}^{\infty} a_{n} \phi(n, t)
$$

By Lemma 5 and the Abel transformation, using (2.5), (2.6) and $s_{n}^{\alpha}=o\left(n^{\alpha}\right)$,

$$
\begin{aligned}
\sum_{n=1}^{\infty} a_{n} \phi(n, t) & =\sum_{\nu=1}^{\infty} s_{\nu}^{\alpha} \sum_{n=\nu}^{\infty} A_{n-\nu}^{\beta-\alpha} \Delta^{\beta+2} \phi(n, t) \\
& =\sum_{\nu=1}^{\infty} \sigma_{\nu}^{\alpha}\left(A_{\nu}^{\alpha} \sum_{n=\nu}^{\infty} A_{n-\nu}^{\beta-\alpha} \Delta^{\beta+2} \phi(n, t)\right) \\
& =\sum_{\nu=1}^{\infty}\left(\sigma_{\nu}^{\alpha}-\sigma_{\nu+1}^{\alpha}\right) U_{\nu}(t),
\end{aligned}
$$

where $\sigma_{\nu}^{\alpha}=s_{\nu}^{\alpha} / A_{\nu}^{\alpha}$. Since the series is evaluable $|C, \alpha|$, that is,

$$
\sum_{\nu=1}^{\infty}\left|\sigma_{\nu}^{\alpha}-\sigma_{\nu+1}^{\alpha}\right|<+\infty \text {, }
$$

using (2.6), for an arbitrary positive number $\epsilon$, there exists an $N=N(\epsilon)$ such that

$$
\left|\sum_{\nu=N}^{\infty}\left(\sigma_{\nu}^{\alpha}-\sigma_{\nu+1}^{\alpha}\right) U_{\nu}(t)\right|=O\left(t^{2 \alpha-1} \sum_{\nu=N}^{\infty}\left|\sigma_{\nu}^{\alpha}-\sigma_{\nu+1}^{\alpha}\right|\right)<\epsilon t^{2 \alpha-1} .
$$

On the other hand, for a fixed $\nu>0$, by (2.3),

$$
\begin{aligned}
\sum_{n=\nu}^{\infty} A_{n-\nu}^{\beta-\alpha} \Delta^{\beta+2} \phi(n, t) & =O\left(t^{\alpha+\beta+1} \sum_{n=\nu}^{\infty}(n-\nu+1)^{\beta-\alpha} n^{-\alpha}\right) \\
& =O\left(t^{\alpha+\beta+1}\right) .
\end{aligned}
$$

Then, for $m \leqq N$,

$U_{m}(t)=\sum_{\nu=1}^{m} A_{\nu}^{\alpha} \sum_{n=\nu}^{\infty} A_{n-\nu}^{\beta-\alpha} \Delta^{\beta+2} \phi(n, t)=O\left(t^{\alpha+\beta+1} \sum_{\nu=1}^{m} A_{\nu}^{\alpha}\right)=O\left(t^{\alpha+\beta+1} N^{\alpha+1}\right)$.

Hence

$$
\lim _{t \rightarrow 0+} \sum_{\nu=1}^{N}\left(\sigma_{\nu}^{\alpha}-\sigma_{\nu+1}^{\alpha}\right) U_{\nu}(t) / t^{2 \alpha-1}=0
$$


Therefore we have

$$
\limsup _{t \rightarrow 0+}\left|\sum_{\nu=1}^{\infty}\left(\sigma_{\nu}^{\alpha}-\sigma_{\nu+1}^{\alpha}\right) U_{\nu}(t) / t^{2 \alpha-1}\right| \leqq \epsilon .
$$

Since $\epsilon$ is arbitrary, we get

$$
\lim _{t \rightarrow 0+} \sum_{\nu=1}^{\infty}\left(\sigma_{\nu}^{\alpha}-\sigma_{\nu+1}^{\alpha}\right) U_{\nu}(t) / t^{2 \alpha-1}=0,
$$

that is,

$$
\lim _{t \rightarrow 0+} \Phi_{\alpha}^{*}(t) / t^{2 \alpha-1}=0,
$$

and the theorem in which $\alpha$ is not an integer is completely proved.

4. Bessel summability. A series $\sum_{n=1}^{\infty} c_{n}$ is said to be evaluable $\left(J_{\mu}\right)$ to zero if the series

$$
\sum_{n=1}^{\infty} c_{n} \psi(\mu, n t)
$$

converges in some interval $0<t<t_{0}$, and if its sum tends to zero as $t \rightarrow 0$. From the argument in $\S 3$, we have, by (2.4),

Corollary. A series evaluable $|C, \alpha|$ to zero is also evaluable $\left(J_{\alpha-1 / 2}\right)$ to zero when $\alpha \geqq 1$.

\section{REFERENCES}

1. L. S. Bosanquet, On the Cesdro summation of Fourier series and allied series, Proc. London Math. Soc. 37 (1934), 17-32.

2. - The absolute Cesiro summability of a Fourier series, Proc. London Math. Soc. 41 (1936), 517-528.

3. K. Chandrasekharan and O. Szász, On Bessel summation, Amer. J. Math. 70 (1948), 709-729.

4. N. Obreschkoff, Über das Riemannsche Summierungsverfahren, Math. Z. 48 (1942), 441-454.

5. C. T. Rajagopal, On the Riemann-Cesdro summability of series and integrals, Tôhoku Math. J. 9 (1957), 247-263.

Chiba Institute of Technology, Chiba, Japan 